\title{
Applications of Expert Diagnosis Learning Defense System with Technology of Cloud Sensors to Enhance the Reliabilities of Machines
}

\author{
Wen-Jie Zheng, ${ }^{1}$ Tzu-Hung Chang, ${ }^{2 *}$ Ming $\mathrm{Li}^{3},{ }^{3}$ and Cheng-Fu Yang ${ }^{4 *}$ \\ ${ }^{1}$ School of Information Engineering, Jimei University, Xiamen, Fujian 361021, China \\ ${ }^{2}$ Department of Electrical Engineering, National Kaohsiung University of Applied Sciences, \\ Kaohsiung 807, Taiwan \\ ${ }^{3}$ School of Electronical and Information Engineering, Xi'an Jiaotong University, Xi'an 710049, China \\ ${ }^{4}$ Department of Chemical and Materials Engineering, National University of Kaohsiung, \\ Kaohsiung 811, Taiwan
}

(Received April 25, 2019; accepted October 18, 2019)

Keywords: expert diagnosis learning defense (EDLD) system, microcontroller unit (MCU), cloud sensors (CS)

The expert diagnosis learning defense (EDLD) system has self-learning capability; thus, it can be used to enhance the fault defense capabilities and improve the reliabilities of machines and equipment. In this study, we designed and fabricated a group of EDLD system modules, that could apply the technology of cloud sensors to achieve the function of automatic defense of failure. If the failure problems of machines and equipment were found by a multifunction sensor and noted immediately, all the operation reliabilities of machines and equipment could be controlled. Even if the numbers of machines and equipment were more than those of engineers, the machines and equipment could also be handled easily. The EDLD system would collect much data (or so-called big data), which were detected by numerous microsystems. Each microsystem was composed of several cloud sensors, one microcontroller unit (MCU) card, and one wireless communication card to achieve the function of multidetection. Each microsystem had its own monitoring regions, and when the detective values of the monitoring regions were beyond the warning or admonished ones, the messages were sent automatically and immediately by the EDLD system, and the engineers would be notified to troubleshoot the problems. The monitoring process is convenient and simple because if the equipment or machines suddenly lose their functions, the EDLD system with the technology of cloud sensors can greatly reduce the loads of the engineers.

\section{Introduction}

Recently, a large number of computer, communication, and consumer electronic (3C) products have been rapidly booming, and they have become smaller in capacity and consume less energy. For that, semiconductor industries must also have sophisticated processes and they are extended to cover the production capacity of high-end products according to the demands

\footnotetext{
*Corresponding author: e-mail: a0926271535@gmail.com

*Corresponding author: e-mail: cfyang@nuk.edu.tw

https://doi.org/10.18494/SAM.2019.2504
} 
of the market. Also, the numbers of machines and equipment in a factory are increasing yearly, which will increase the importance and necessity of using automatic machines and equipment. However, most functions of automatic machines and equipment are limited in fully automatic production lines. There are no automatic perception and detection systems to prevent errors and enhance the error defense capabilities of machines and equipment, which can improve their reliabilities in advance.

The fourth industrial revolution (Industry 4.0) is a hot issue in terms of smart manufacturing owing to the considerable advancements of many research fields related to computer science. However, the cloud platform can be used to handle the diversity data of machines and equipment and provide a unified analytics layer such that meaningful information can be extracted by mining these data. ${ }^{(1)}$ For real-time monitoring and detection, cloud sensing, data collection, and computing have played important roles. Wireless or cloud sensors used in wireless sensor network (WSN) scenarios can be developed with a large number of low-cost tiny sensor nodes to apply them in specific areas. Sensor nodes can sense a large amount of information from the environment and route them to a basic station. At the basic station, the collected information are aggregated, analyzed, and finally used by different applications. ${ }^{(2)}$

Cloud sensors can provide a low-cost system for monitoring the signals or data from remote sensors. They can also incorporate a relay suitable for closing the observatory dome or activating other equipment when cloud sensing is achieved and the relay operates independently of the computer. The big data of the expert diagnosis learning defense (EDLD) system can collect a large amount of information using numerous microsystems, and the method of designing the EDLD system is as shown in Fig. $1 .{ }^{(3-7)}$ Each microsystem must be equipped with a few cloud sensors, a microcontroller unit $(\mathrm{MCU})$ card, ${ }^{(8)}$ a power supply with an integrated circuit board, and a wireless communication card (WCC) to achieve multiple monitoring functions. Figure 2 shows the top view of the architecture of the investigated microsystem.

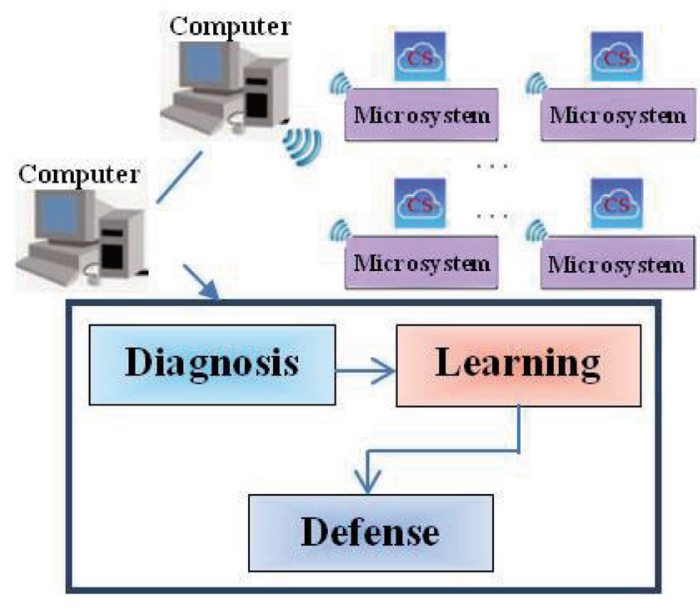

Fig. 1. (Color online) Proposed EDLD system for collecting big data.

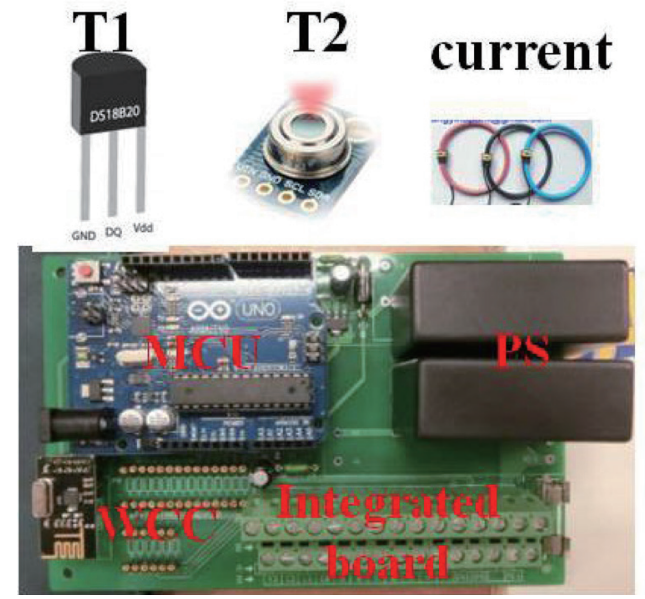

Fig. 2. (Color online) Top view of the designed microsystem. T1, temperature 1; T2, temperature 2 . 
Concerning the use of MCU cards, there were 37 sensors compatible with the cloud sensors and MCU module. In this study, we only used six sensors to construct the EDLD system. The selected six different sensors were used to sense temperature, voltage (AC and DC), current, magnetic field, pressure, and leakage current. When the error data was sensed by the six sensors, the sensors would drive the warning signals, which were transmitted by the MCU module to the local server in the wireless communication module. The Supervisory Control and Data Acquisition (SCADA) system is an architecture of the control system that uses computers, networked data communications, and graphical user interfaces for high-level supervisory management. However, the SCADA system uses other peripheral devices such as a programmable logic controller (PLC) and discrete proportional-integral-derivative (PID) controllers as the interface to connect with plants and machineries.

When the subsystems of the networks were constructed, the warning signals (big data) could provide feedback to the monitoring center, which is a server computer. The big data could be found in the SCADA system, which could be used to immediately monitor the operation conditions of equipment and machines. After that, if the equipment and machines could not operate normally, we would use the EDLD system to analyze the failure information obtained from the equipment and machines and generate a new monitoring rule. Because we could update the signals of the MCU microsystems with new monitoring rules, the interactive model of the EDLD system could make them have a high learning capability. Thus, more accurate predictions (to determine if the warning signals or error messages are correct) could be obtained, and faulty or failed machines and equipment could be immediately found and recognized. This means that the EDLD system can interact with engineers and reduce the probabilities of sudden failures of machines and equipment, thus improving the reliabilities of the machines and equipment, as shown in Fig. 3.

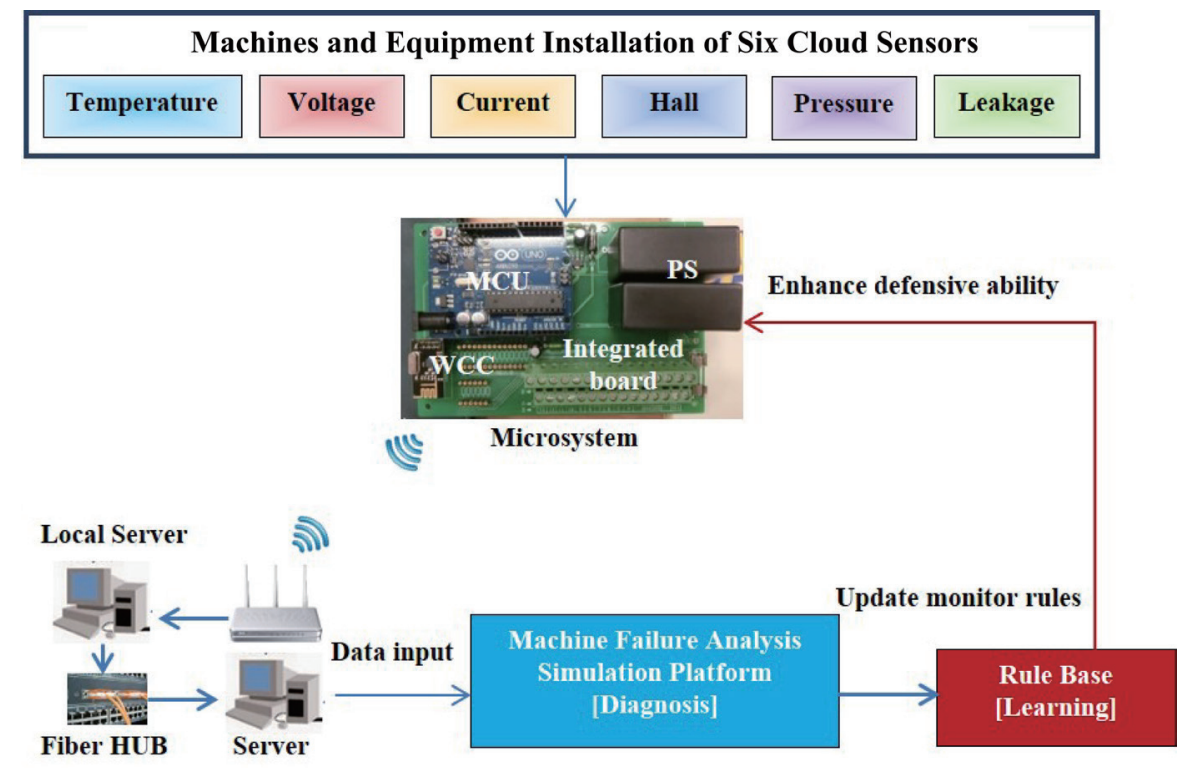

Fig. 3. (Color online) Architecture diagram of the designed EDLD system. 


\section{Methodology}

In this research, we used a large number of cloud sensors for monitoring machines and equipment, and then collected a large number of warning signals from them. These data were managed by the designed EDLD system to achieve the message diagnosis, determining if the warning signals or error messages were correct. The EDLD system had the capability of learning, and the monitoring rules in it could be updated. These data could be used to construct a database and determine the warning signals and enhance the preventive capabilities of the monitored machines and equipment. Thus, we can easily improve the reliabilities of online machines and equipment. To achieve the scheduled function, the following methodology is used to investigate the learning capability of the EDLD system.

\subsection{Expert system}

An expert system is a computer system that emulates the decision-making capability of a human expert and is suitable for different tasks, such as diagnosing, interpreting, monitoring, forecasting, planning, and designing tasks. An expert system has the first truly successful forms in artificial intelligence (AI) software. However, the expert system is not part of true AI software since it lacks the capability to learn autonomously from external data. For example, inaccurate data, incomplete information, and shortages of expert and human expertise cannot be recognized as theories and methods. An expert system can bring significant economic benefits for its users, and thus, the task of solving the general expert system is a knowledgeintensive work. For example, an expert system can carry out these tasks using more efficient and economical methods without experienced experts and can greatly reduce the amount of labor force and training costs. Because the software of an expert system is easy to duplicate, it can extensively disseminate expert knowledge and experiences, and increase the efficiencies of the finite and expensive skills and professional knowledge.

Also, an expert system is recognized as an important branch of AI software and can be regarded as a type of computer intelligent program system with specialized knowledge and experience. The expert system can generally be used to simulate the complexities of knowledge, which can usually be used to solve these problems by experts who specialize in the normal knowledge and in the reasoning knowledge of AI technology. In general, an expert system is equal to knowledge base + reasoning machine, so an expert system is also known as a knowledge-based system. A proposed expert system must have elements as follows:

a. Expert-based diagnosis knowledge

Expert-based diagnosis knowledge is constructed with the experience sharing of some experienced or expert engineers. We then used the rules of experience expert-based knowledge as references to write a program.

b. Learning rule bases

We analyzed and determined the reason for the failure of each device after we received the indexes of the monitoring warning signals, and then modified the accumulating data to achieve the benefits of learning. In fact, in the process of accumulating data, we should 
establish the strongest monitoring base rule, and then the signals could provide feedback to the machines and equipment for making correct controls. After we constructed the base rule to "teach" the machines and equipment for learning, we then used it to form the architecture of the optimal defense strategy, which could avoid the machines and equipment to be stopped by the error messages.

\subsection{Microsystem and cloud sensors}

The investigated microsystem was a combination of an MCU \{using Arduino Promini [Fig. 4(a)] or Arduino Uno [Fig. 4(b)]\}, an integrated circuit board, and a WCC, where nRF24L01 [Fig. 4(c)] or ESP8266 [Fig. 4(d)] was selected as the WCC. Cloud sensors are the devices that can be installed in the machines and equipment for monitoring, and can also contain the signals and messages via the microsystem sensors' reading and monitoring statuses, where the signals and messages can be transferred by the wireless module to a local PC. The wireless transmission sensors can monitor the statuses of machines and equipment, and obtain the signals and messages in time. These sensors can be installed without connection with the wired network, so they are then called cloud sensors.

After we learned the definitions and specifications of the microsystems and cloud sensors, we began to use and build them as the system architecture for sensing the failures of equipment and machines. First, we laid out and constructed the installed microsystems and cloud sensors on the machines and equipment that were going wrong, and we also drove machines and equipment with the monitoring rules programmed on the MCU card. Then, the wireless

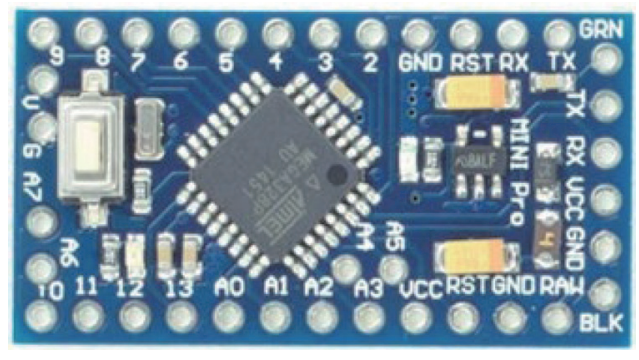

(a)

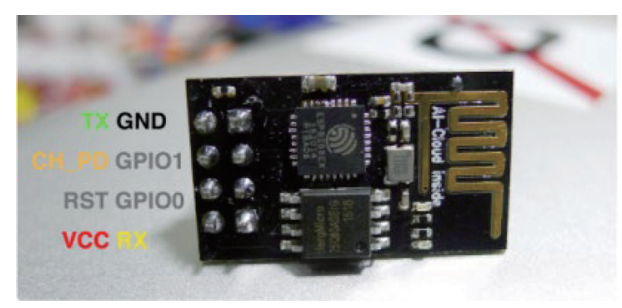

(c)

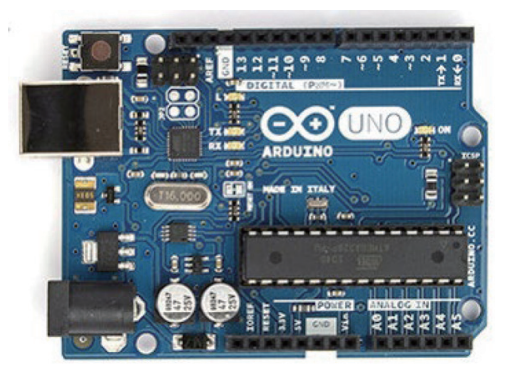

(b)

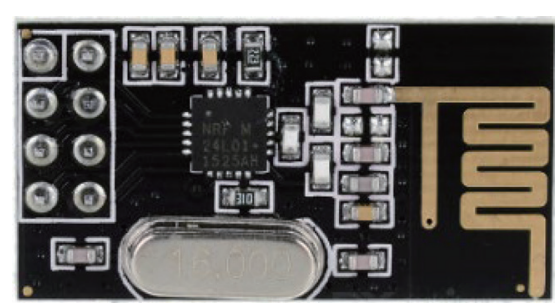

(d)

Fig. 4. (Color online) Surface morphologies of (a) Arduino Pro-mini, (b) Arduino Uno, (c) ESP8266, and (d) nRF24L01. 
transmission modules read and collected the messages and data from the cloud sensors, and transmitted them to the local PC using the wireless transmission system. Finally, via the constructed network, the messages and data were sent back to the center server immediately for monitoring the statuses of machines and equipment, and carrying out the predictive analysis by the expert system.

\section{Fault Prediction Expert System}

In this study, we propose four different systems to describe how we used the fault prediction expert system as the cloud sensor to enhance the reliabilities of machines and equipment.

Case A: Playing-up or fast-fault components of variable-frequency drive (VFD) for failure prediction using an expert system

As shown in Fig. 5, the microsystems and cloud sensors were installed in the eight groups of VFD control panels to monitor the operations of the playing-up or fast-fault components, including the capacitor temperature detection at the power source side, the DC output voltage detection of the power supply, and the temperature detection of the VFD internal components. Some components with the sensing function were attached to the VFD control panels and detected the playing-up or fast-fault conditions as signals. The alarm-signal settings of each component were based on the fault prediction expert system that could learn the functions after obtaining the parameters several times to finish the learning process. In this manner, we constructed a customized monitoring system that could have the optimal function to monitor the system. After the fault prediction expert system was installed, the warning signals were

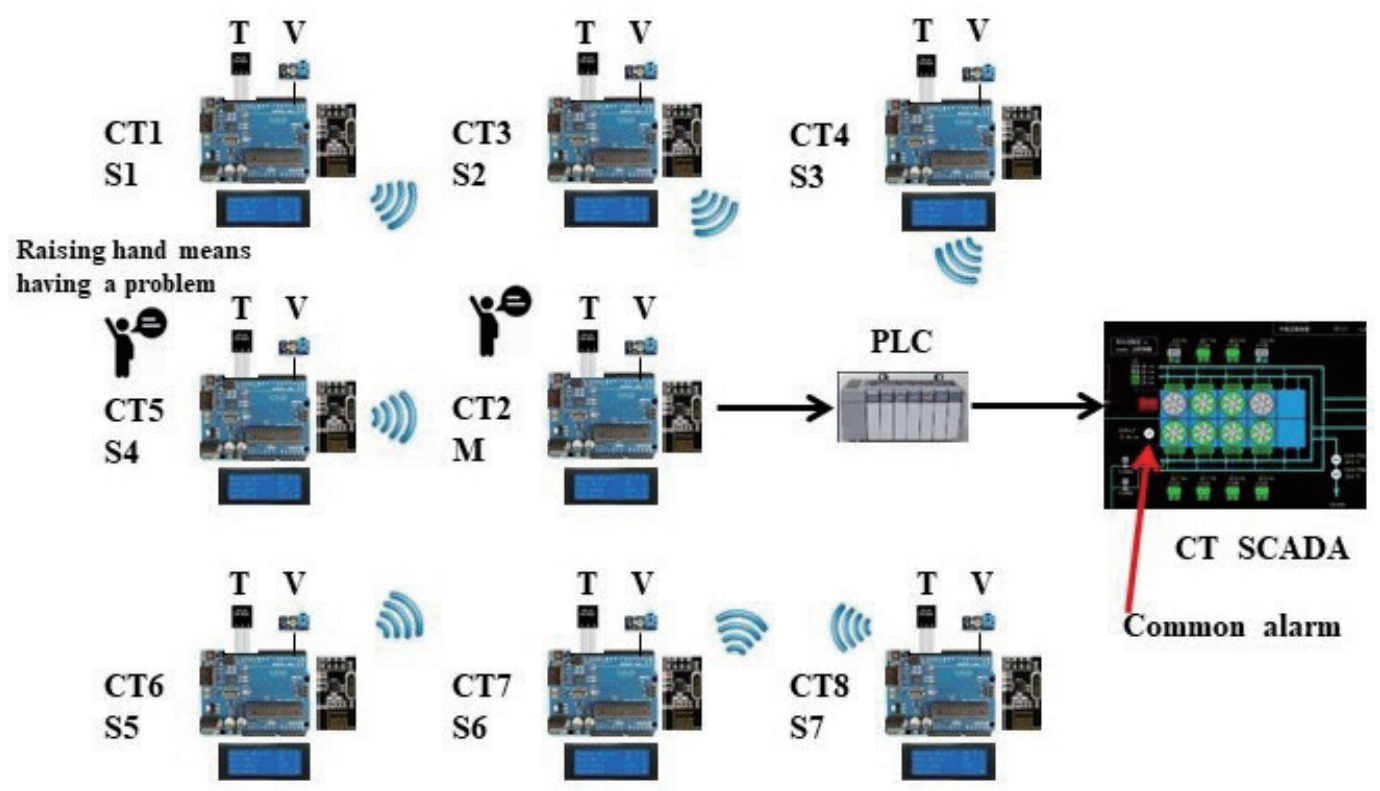

Fig. 5. (Color online) Architecture of the designed EDLD system with eight sets of VFD. T, temperature; $\mathrm{V}$, voltage. 
detected before the failure signals, and they were replaced by new products for normal sensing processes. Because all things were in control, the problems of sudden failures caused by overloading were minimized.

\section{Case B: Wire-alarming system for prediction of fire in a distribution box}

As shown in Fig. 6, the microsystems and cloud temperature sensors were installed in a distribution box to monitor the temperature of the internal wires. If the operating temperatures were reached and higher than the set values, the alarm messages would automatically be sent via the Wi-Fi modules to a remote server. The remote server would send a message to cell phones' application software (APP) as a notification. Then, the engineers would know that the wire temperature of the distribution box was very high and that there was a risk of fire. Then, the engineers could immediately deal with the problem and find a method to prevent fire in the distribution box. Even the microsystems and sensors are very simple devices, they can be installed in the distribution box as a wire-alarming system to prevent fire.

Case C: Fault prediction of dynamic insulation deterioration in a transformer by the expert system

As shown in Fig. 7, a contactless Hall element was installed a transformer that was specifically located between the winding coil and the ground to detect the strength of the magnetic field. When the magnetic field was very large, the insulation resistance started to degenerate. When it was smaller than the warning values, the fault prediction expert system immediately reflected and dealt with the response, and the transformer would not lead to a relatively short circuit and burn because the high current and temperature had been sensed.
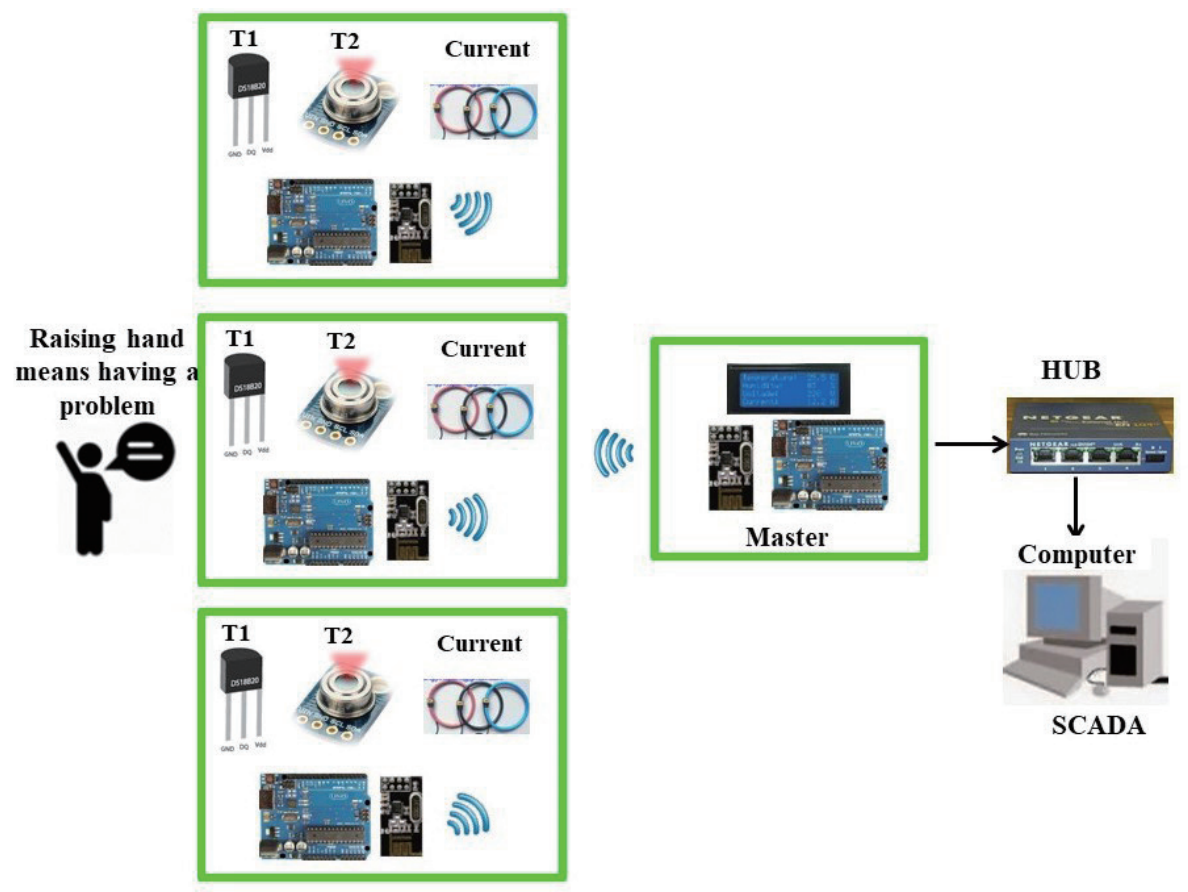

Fig. 6. (Color online) Wire-alarming system for fire in a distribution box. 


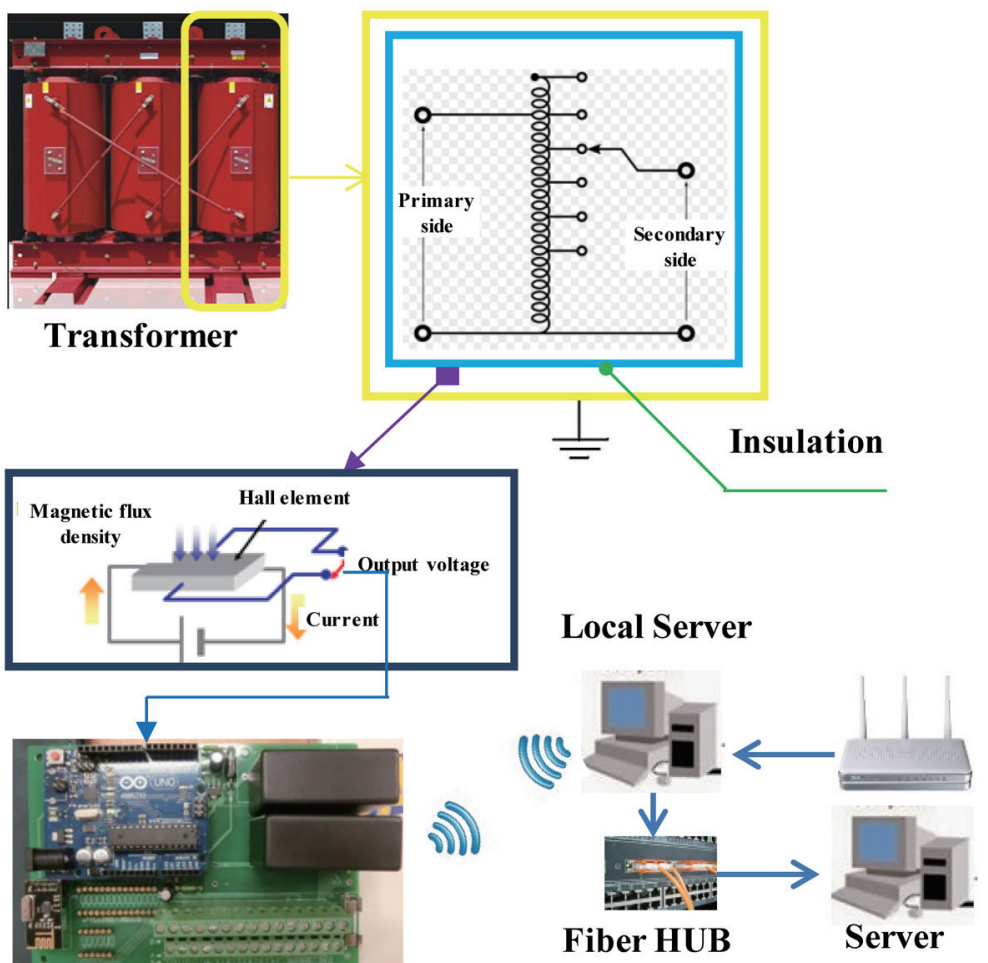

Fig. 7. (Color online) Dynamic insulation deterioration of transformer.

Case D: Preventing the fast-fault components of a circuit board from burning using the fault prediction expert system

The system was investigated to detect the temperatures of the devices on the electronic or printed circuit board (PCB). If the temperatures of the devices on the PCB reached the warning values, the sensors would immediately send the information to notify the engineers. If the problem of high temperature could not be dealt with immediately, this would lead to a short circuit and the burning of the PCB.

Figure 8 shows the constructed EDLD system to be used in the cooling system of a cooling tower. This constructed system had different detection functions, could detect the voltage of the power supply, and had a VFD subsystem. The subsystem had the early warning function and contained an insulated gate bipolar transistor (IGBT) used as the temperature sensor, a DC capacitor temperature sensor with an automatic monitoring function, a filter capacitor used as a temperature sensor, and $\mathrm{P} / \mathrm{S}$ used as a voltage detector, as shown in Fig. 8. We used an Arduino-based integrated circuit board ( $\mathrm{MCU}+\mathrm{RF}$ wireless model + power) to transmit the signals and data obtained from the slave station (personal computer) to the master station by wireless communication. These signals included early warning signals and data, which were generated from the sensors of the IGBT, DC capacitor, and filter capacitor, and the $\mathrm{P} / \mathrm{S}$ voltage detector. 


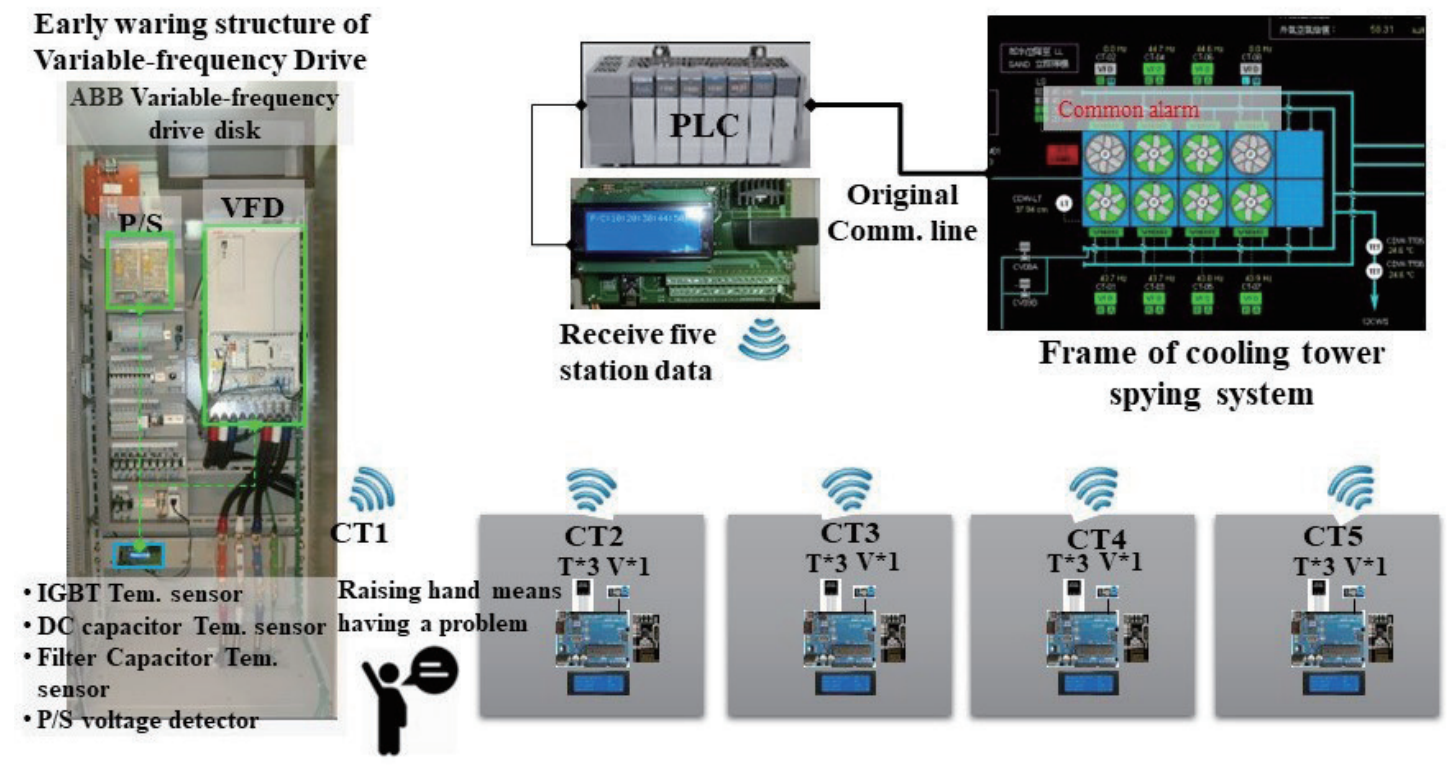

Fig. 8. (Color online) Constructed EDLD system to be used in the cooling system of a cooling tower with different detective functions.

The architecture for this system is that the master station would connect with the PLC system, and finally, we would install "common early warning" on the SCADA to display the sensing results. Also, the system in Fig. 8 could merge with the existing SCADA system, could have the function of early warning for the failures of devices, and could enhance the fault selfprotection capabilities of devices. Because wireless communication is the main method for data transmission, the benefits of this system are that we can reduce the manpower to construct the network by $90 \%$, reduce the loading of the communication system by $90 \%$, and reduce the numbers of Arduino devices by about $50 \%$.

\section{Conclusions}

In this study, the EDLD system was used to enhance the fault defense capabilities and improve the reliabilities of the machines and equipment. The EDLD system would collect much data from numerous microsystems, which were composed of several cloud sensors, one MCU card, and one WCC to achieve the function of multidetection. We proposed four methods of constructing the fault prediction expert system, including the use of the playing-up or fastfault components of VFD, the use of a wire-alarming system for the prediction of fire in a distribution box, the fault prediction of dynamic insulation deterioration in a transformer, and the use of the playing-up or fast-fault circuit board components. When the constructed EDLD system was used in the cooling system of a cooling tower, we could reduce the manpower to construct the network by $90 \%$, reduce the loading of the communication system by $90 \%$, and reduce the numbers of Arduino devices by about $50 \%$. 


\section{Acknowledgments}

This work was supported by projects under Nos. MOST 108-2221-E-390-005 and MOST 108-2622-E-390-002-CC3.

\section{References}

1 J. Dutta, S. Roy, and C. Chowdhury: Microsyst. Technol. 25 (2019) 83.

2 A. Ray and D. De: Microsyst. Technol. 23 (2017) 4307.

3 J. S. Wu, C. C. Liu, K. L. Liou, and R. F. Chu: IEEE Trans. PAS-12 (1997) 69.

4 C. Anantaram, P. Joshi, K, Deshpande, and P. Trivedi: Proc. 9th Conf. Artificial Intelligence for Applications (1993) 63

5 H. J. Lee and Y. M. Park: IEEE Power Eng. Review 17 (1996) 49

6 C. L. Yang and A. Yokoyama: Proc. 4th Symp. Expert System Application to Power Systems (1993) 321.

7 S. J. Lee, C. C. Liu, and S. S. Venkata: IFAC Proc. 21 (1998) 423.

8 C. C. Liu, S. J. Lee, and S. S. Venkata: IEEE Trans. Power Systems 3 (1998) 619. 